\title{
EXTERNALIDADES ASOCIADAS A LA DISTRIBUCIÓN DE ALIMENTOS PERECEDEROS EN LA ÚLTIMA MILLA
}

\author{
PATRICIA JIMÉNEZ BARRANTES \\ Universidad Estatal a Distancia, Costa Rica \\ mpatjb@yaho.com.mx
}

\begin{abstract}
JORGE M. FONSECA LAURENT
Organización de las Naciones Unidas para la Alimentación y la Agricultura (FAO), Italia

jorge.fonseca@fao.org
\end{abstract}

\section{RESUMEN}

Este artículo propone una herramienta, sustentada en el análisis financiero, para estimar los costos de dos externalidades relacionadas con la distribución de alimentos perecederos en la denominada "última milla" en el contexto urbano: i) la contaminación por combustión y consecuente emisión de CO2, atribuible a las flotas de transporte de carga que distribuyen los alimentos frescos en las ciudades y ii) el desgaste que esa flota vehicular provoca en el mantenimiento preventivo de las carreteras de las ciudades de Sacramento y Baton Rouge, Estados Unidos; San José, Costa Rica; Santa Marta, Colombia; Liguria, Italia; Nottingham, Reino Unido; Adelaida, Australia; Lianjiang, China y Jos, Nigeria. Este costeo se plantea a partir de los precios implementados por la Comunidad Europea bajo el Sistema de Comercio de Derechos de Emisión, de impuestos designados en países como Australia y China y del pago por servicios ambientales por captura de carbono en Costa Rica.

PALABRAS CLAVE: EXTERNALIDADES, CONTAMINACIÓN CON CO2, MANTENIMIENTO PREVENTIVO DE CARRETERAS, PRODUCTOS PERECEDEROS, ÚLTIMA MILLA.

\section{ABSTRACT}

This study propose a tool based in financial analysis to estimate the costs of two externalities related to the distribution of perishable foods in the so called "last mile" in the urban context: i) $\mathrm{CO} 2$ pollution by its combustion and consequent emission that are attributable to the light freight fleet that distribute fresh foods in the cities, and ii) the decay that this vehicular fleet causes in the preventive maintenance of the roads of the cities of Sacramento and Baton Rouge, United States; San José, Costa Rica; Santa Marta, Colombia; Liguria, Italy; Nottingham, United Kingdom; Adelaide, Australia; Lianjiang, China and Jos, Nigeria. The cost analysis is based on prices implemented by the European Community under the emissions trading system, pollution taxes designated by countries such as Australia and China and payment for Environmental Services for carbon sequestration in Costa Rica.

KEY WORDS: EXTERNALITIES, CO2 POLLUTION, ROAD PREVENTIVE MAINTENANCE, FRESH FOOD, LAST MILE. 


\section{INTRODUCCIÓN}

Para el desarrollo del tema de costeo de dos externalidades, específicamente, las emisiones de CO2 por combustión asociadas a la distribución de alimentos frescos y el desgaste en el mantenimiento de las carreteras en la última milla, se seleccionaron nueve países que representaran a los cinco continentes. Estos poseen características similares en cantidad de la población; estructuras de gobierno que contempla a las municipalidades, comunas o gobiernos locales; gobiernos locales que tengan actividades comunes, relacionadas con la distribución de alimentos frescos en las ciudades.

Se proponen dos objetivos: (1) Analizar las actividades logísticas que realizan los gobiernos locales relacionadas con la distribución de alimentos perecederos en la última milla, en una o dos ciudades por continente. (2) Determinar una herramienta financiera para estimar los costos de las externalidades que impactan a los gobiernos locales asociada a la distribución de alimentos en la última milla.

El artículo se divide en cuatro secciones. En la primera, se describen los fundamentos teóricos, los conceptos de diferentes autores en los temas relacionados con la última milla, externalidades, dinámicas de las ciudades, logística urbana, logística del transporte urbano y costos de la contaminación ambiental. La segunda sección se dedica a la metodología utilizada, que consistió en el método de investigación cuantitativa alimentada con los siguientes datos: (i) Estadísticas derivadas de la hoja del consumo de frutas y verduras de la base de datos de la Organización de las Naciones Unidas para la Alimentación y la Agricultura (FAO) conocidas como FAOSTAT; (ii) datos de la contaminación por CO2 de la Internacional Energy Agency (IEA) para los sectores eléctrico, manufactura, transporte y otros; (iii) información sobre las poblaciones de países y ciudades en estudio de la Organización de Na- ciones Unidas (ONU); (iv) datos del producto interno bruto (PIB) del Banco Mundial (BM); (v) estadísticas de población, flotas vehiculares por registro e inflación de las bases de datos cada país y variedad de fórmulas para la construcción de la matriz como herramienta financiera de costos de las externalidades.

La tercera sección presenta los resultados del análisis, cálculos de costos de las externalidades de contaminación por CO2 asociada a los vehículos de transporte de alimentos en la última milla y el costo del mantenimiento preventivo de las carreteras de las ciudades. Finalmente, en la última parte, se describen las lecciones aprendidas de la investigación como conclusión.

\section{FUNDAMENTOS TEÓRICOS}

La fundamentación teórica se sustentó en diversos autores que abarcan la contaminación ambiental como una externalidad concreta y sus propuestas para ofrecer un costo económico. En su mayoría, están basados en algunos supuestos, así como la terminología de la última milla en las ciudades, su dinámica y los cambios en la logística urbana.

\section{La última milla urbana}

Hay una verdad inminente, la población urbana del mundo pasó del 43.53\% en el año 2000 al $53.85 \%$ en el año 2015. Se trata de un aumento de 10 puntos porcentuales lo cual, claramente, es un indicador de la expansión de las ciudades y de la migración de los pobladores hacia las zonas urbanas (BM, 2015). Esta realidad de aumento en la zona urbana viene acompañada de oportunidades de mejora en aspectos metodológicos de la planificación y la logística urbana, considerando la mitigación de las externalidades negativas.

Rodrigue, Claude y Slack (2013) indican que la última milla 
ha generado mucha atención debido a la importancia que tiene transportar los bienes y servicios de los mayoristas al consumidor final donde se da la mayor interacción de personas y empresas, creando mayor congestión, requiriendo mayor seguridad, espacios de interacción comercial. Los problemas de la última milla siguen siendo prevalecientes para la distribución de mercancías en las áreas urbanas, donde la congestión es comúnmente ligada a una disminución en la frecuencia de las entregas de mercancías sin tener la capacidad de garantizar un servicio similar (p. 220).

\section{Externalidades}

Las externalidades en los sistemas productivos, de acuerdo a Vázquez (2014), se definen como:

Decisiones de consumo, producción e inversión que toman los individuos, los hogares y las empresas y que afectan a terceros que no participan directamente en esas transacciones. Cuando hay externalidades se desencadenan efectos indirectos que repercuten en las oportunidades de consumo y producción de terceros, pero el precio del producto no refleja esas externalidades. Cuando se produce una ventaja gratuita o una desventaja, un perjuicio sin compensación monetaria estamos hablando de una externalidad (p. 2).

Asimismo, Delacámara (2008) reflexiona sobre significado de las externalidades a partir de la incógnita:

¿Qué hacer ante la evidencia de una externalidad? La sugerencia del análisis económico parece clara: en primer lugar, reconocer su existencia. Sólo entonces se puede plantear el sentido de estimarla por procedimientos más o menos sofisticados, según el caso. El análisis de externalidades arroja beneficios en el proceso ya que introduce transparencia en la gestión, se enriquece la información sobre las actividades económicas objeto de análisis, se reduce la discrecionalidad de algunas decisiones. Aunque uno no fuese capaz de estimarlas, por desconocimiento técnico o por escasez de medios, reflexionar sobre ellas ayuda, en cualquier caso (p.13).

Los autores nos presentan el término de externalidad como un evento de intercambio de bienes cuyo precio no contempla los beneficios o efectos indirectos que afectan a terceros.

\section{La logística urbana}

Otro reto muy importante son los cambios a los que la planificación urbana se enfrenta. Para Taniguchi (2014):

La importancia de la planificación del transporte urbano y el uso de la tierra son los componentes de la eficiencia urbana. Todas las actividades de la ciudad aunadas al tránsito vehicular particular y de carga, pone en las urbes un gran reto ante el conflicto de intereses por los recursos públicos como la seguridad, aire, ruido ambiental, agua, desechos sólidos en las que los habitantes y empresas participan (p. 310).

La logística urbana en el transporte de carga en las ciudades que tiene posiciones de conflicto con los intereses de las acciones de la administración pública y las diferentes actividades económicas de los centros urbanos, considerando las externalidades causadas por la dinámica de las ciudades (Russo y Comí, 2011, p. 718).

La logística urbana la define Taniguchi (2014) como "el proceso de optimizar totalmente las ac- 
tividades privadas de transporte y logística en las áreas urbanas considerando el tránsito, la congestión y el consumo de energía dentro de la estructura de una economía de mercados" (p. 311).

\section{Dinámica de las ciudades}

La dinámica de las ciudades se enfrenta a un cambio radical de la planeación logística de estas, un aumento en la población que requiere ser satisfecha en dos aspectos. Primero, necesidades básicas de los habitantes (vivienda, salud, educación, alimentación) y las actividades que realizan, (salir de compras, actividades al aire libre, realizar algún deporte). Segundo, las actividades de empresas comerciales que requieren de espacios para el comercio, parqueos y rutas de transporte de entrega de mercancía.

Como aporte a la solución en la dinámica de las ciudades Taniguchi (2014) propone tres elementos esenciales para promover la logística en la ciudad:

(i) Aplicación de tecnologías innovadoras de las TIC (Tecnologías de la Información y la Comunicación) e ITS (Sistemas Inteligentes de Transporte), (ii) cambio de mentalidad de gerentes de logística, y (iii) asociaciones público-privadas. La aplicación de tecnologías innovadoras TIC y de ITS en el transporte urbano de mercancías, permite recolectar datos precisos (de tiempos y movimientos) de los camiones en la recolección y entrega en las carreteras urbanas, con costos más bajos. Los datos digitales se pueden utilizar para optimizar la planificación de la ruta del vehículo y los horarios de una manera dinámica y estocástica. Este tipo de optimización de las operaciones de los vehículos puede contribuir a la reducción de los costos logísticos, disminuyendo las emisiones de CO2, NOx y SPM así como aliviar la congestión del tráfico. Por lo tanto, las empresas privadas como la sociedad en general pueden beneficiarse de la aplicación de tecnologías innovadoras TIC e ITS en términos de eficiencia de la logística, así como reducción de los impactos ambientales negativos (p. 311).

\section{Costos de contaminación por $\mathrm{CO} 2$ sector transporte carga}

Azquela (como se citó en Reyes, Galván y Aguiar, 2005), define que "un costo externo o externalidad negativa existe cuando se dan las dos condiciones siguientes: 1) Una actividad de un agente económico provoca una pérdida de bienestar a otro agente, y 2) la pérdida de bienestar no está compensada" (p. 438).

Los costos de la contaminación normalmente están expresados en términos macroeconómicos; o sea, hasta que el precio o valor marginal del producto iguale al costo marginal.

En 1920, Arthur Pigou propuso, para las emisiones que estaban contaminando el ambiente, una la modalidad de impuesto en términos económicos como la figura de un precio al derecho a contaminar.

Pearce y Turner (citados en Reyes et al., 2005) definen "el impuesto pigouviano óptimo como aquel igual al costo marginal externo en otras palabras al daño causado por una unidad marginal de contaminación en el nivel óptimo de contaminación" (p. 439). El costo financiero de la contaminación ambiental toma los precios dados en el mercado, como la tasa impositiva por emisión, el precio del documento comercial por emisión o el incentivo por captura de carbono, para el cálculo del mismo, este mecanismo flexible se utilizó para el cálculo de la proyección financiera de costos en la distribución de alimentos frescos en la última milla.

Díaz (2012) plantea un cálculo matemático que toma como base los datos país de Alemania para comparar con los países de estudio y cuantificar: 
(...) las pérdidas de incapacidad laboral ocasionados por enfermedades respiratorias, las pérdidas de recursos humanos por muertes, los costos de rehabilitación de las personas afectadas por enfermedades respiratorias, el costo de las enfermedades cardiovasculares, y el costo de reparación de estructuras y edificios como consecuencia de la contaminación atmosférica (p. 38).

Delacámara (2008), en su guía para decisores, indica:

Si bien es cierto el costeo de externalidades ambientales normalmente se basa en propuestas de modelos económicos, no hay ningún enfoque que se esté utilizando con un rango de certeza importante. También se puede recurrir a valores publicados en diferentes meta-análisis desarrollados por prestigiosos autores en este campo a partir de modelos de simulación que predicen el daño causado, no obstante, presentan limitaciones asociadas con la incertidumbre de cualquier resultado que podría invalidar los resultados. Está claro que los valores propuestos están sujetos a numerosas limitantes (p. 52).

\section{Costo de mantenimiento preventivo carreteras}

De acuerdo con Menéndez (2003):

Durante varias décadas, en la mayoría de los países latinoamericanos se consideró que la función primordial de los organismos del Estado responsables de los caminos era construir caminos con los recursos presupuestales asignados. La eficiencia de tales organismos se medía en el número de kilómetros construidos y en el tipo de construcción utilizada; en cambio, la conservación de los caminos ya construidos tuvo un rol secundario. En general, las personas entienden que el camino al haber sido construido con recursos del Estado es de su propiedad y, por lo tanto, el mantenimiento también es de su responsabilidad. Sin embargo, de acuerdo a la legislación vigente, en la mayoría de países, la red vecinal está bajo responsabilidad de los municipios y, en algunos casos, la red secundaria ha sido entregada a la gestión de los gobiernos provinciales o regionales (p. 13).

\section{METODOLOGÍA}

De acuerdo con el marco teórico expuesto, el desarrollo del segmento metodológico se basó en la investigación cuantitativa mediante el uso de estadísticas. Lo anterior para establecer patrones de comportamiento en una población y el desarrollo de actividades productivas perecederas.

\section{Instrumentos}

La investigación se alimentó con las bases de datos estadísticos como FAOSTAT para la selección de frutas y vegetales frescos, montos de producción, de importaciones y de exportaciones de los vegetales y frutas frescas, para determinar el consumo per cápita de los países en estudio. También, datos del Banco Mundial en cuanto al PIB, la inflación, poblaciones totales y urbanas de los países. Además, el IEA, suministrando los datos de emisiones de CO2 por combustión en el sector transporte carretera. Se suman a esta lista los datos de la ONU en población de países y ciudades de estudio. Por último, estadísticas de cada país de las flotillas vehiculares de carga.

\section{Participantes del estudio}

Se consideraron nueve ciudades en los cinco continentes. Estas se seleccionaron de acuerdo con las siguientes características: cantidad de población (500 mil a 2 millones de habitantes); que se cuente con un régimen municipal vigente; que los gobiernos locales tuvieran, por 
lo menos, dos externalidades relacionadas con la distribución de perecederos; que las ciudades estuvieran tanto en la costa como en los valles.

Las características expuestas determinaron los países seleccionados. No se utilizó ningún método aleatorio por la necesidad de contar con información tan específica. Por tanto, los países y ciudades seleccionados fueron: Sacramento y Baton Rouge (Estados Unidos, US), San José (Costa Rica, CR), Santa Marta (Colombia, CO), Notthingham (Reino Unido, UK), Liguria (Italia, IT), Adelaide (Australia, AU), Jos (Nigeria, NG) y Lianjiang (China, CN).

En ninguno de estos países o ciudades existe información de costos de las externalidades propuestas. Esto porque no hay evidencia de costos de la contaminación ambiental en el sector transporte carga de alimentos frescos, ni de los costos asociados al mantenimiento de las carreteras de las ciudades, provocado por los vehículos que distribuyen los alimentos perecederos en la última milla.

\section{Procedimiento de cálculo}

Para la construcción de la herramienta financiera se consideraron diferentes fórmulas para el cálculo monetario, todas ellas se enumerarán a continuación.

- Consumo per cápita en kilos de alimentos frescos. Este dato se requiere para proyectar el consumo en la ciudad de estudio y de la última milla en donde debe ser distribuido.

- Cálculo de la densidad de población.

- Cálculo población de la última milla. Este dato evidencia la cantidad de habitantes a los que se les debe satisfacer sus necesidades de productos frescos. No se considera la población laboral, ni las personas de paso por la ciudad.
- Cálculo de consumo de alimentos, en kilos, en la última milla de la ciudad de estudio por año.

- Capacidad del vehículo de carga por viaje en cantidad de cajas de alimentos frescos, por día.

- Cálculo de vehículos para la distribución alimentos última milla, por año.

- Cálculo de las emisiones por vehículo.

- Cálculo de emisiones por vehículos de carga en la ciudad.

- Cálculo del Incentivo país base.

- Cálculo del Incentivo por país sin programa de incentivos. Con los datos anteriores se calculó el monto monetario por las emisiones en la distribución de alimentos en la última milla.

- Cálculo de datos globales contaminación. Para este cálculo, se requieren los siguientes datos a nivel de país: superficie, emisiones, población, producto nacional bruto per cápita para obtener el factor de relación de la contaminación ambiental, este se compara con el país base en este caso Alemania. El segundo método de cálculo, considerando variables como la salud, ausentismo laboral entre otros.

Los siguientes datos son para el cálculo de la segunda externalidad propuesta:

- Cálculo del recorrido del primer y segundo anillo, adicionando cuatro rayos de dos kilómetros cada uno, para el cálculo del recorrido alrededor de la ciudad y el área de distribución en la última milla.

- Cálculo del costo mantenimiento rutinario vial en las carreteras. 
Con la información anterior se elabora la matriz como herramienta financiera de costos de las externalidades por emisiones de $\mathrm{CO} 2$ por combustión asociadas a la distribución de alimentos frescos y desgaste en el mantenimiento de las carreteras en la última milla.

\section{FÓRMULAS DE CÁLCULO UTILIZADAS}

- Externalidad contaminación de emisiones de CO2 por combustión asociadas a la distribución de alimentos frescos en la última milla.

(i) Cálculo del consumo per cápita de alimentos frescos de cada país por año

$$
\begin{aligned}
& Q P+Q I-Q E=Q O D \\
& A A+S+P e=U E \\
& Q O D-U E=T O D \\
& \text { TOD/PO }=\text { CperC }
\end{aligned}
$$

Donde:

$\begin{array}{ll}\text { QP } & \text { Cantidad Producción } \\ \text { QI } & \text { Cantidad importada alimentos frescos } \\ \text { QE } & \text { Cantidad exportada alimentos frescos } \\ \text { QOD } & \text { Cantidad oferta doméstica } \\ \text { AA } & \text { Alimentos para animales } \\ \text { S } & \text { Semillas } \\ \text { Pe } & \text { Pérdida } \\ \text { UE } & \text { Utilización de elementos } \\ \text { TOD } & \text { Total Oferta doméstica } \\ \text { CperC } & \text { Consumo per cápita }\end{array}$

(ii) Cálculo de densidad de población

$$
P C / S=D
$$

Donde:

PC Población de la ciudad

S Superficie km2 ciudad

D Densidad población

(iii) Cálculo población de la Última milla

$$
S^{*} \mathrm{D}=\mathrm{PUM}
$$

Donde:

S superficie última milla km

D densidad de población ciudad

PUM Población Última Milla

(iv) Cálculo de consumo de alimentos en kilos en la última milla de la ciudad de estudio por año

$$
\mathrm{PUM}^{*} \mathrm{Cpc}=\mathrm{CUM}
$$

Donde:

PUM Población Última Milla

CperC Consumo per cápita en Kg

CUM Consumo/kg Última milla

(v) Capacidad del vehículo de carga por viaje en cantidad de cajas de alimentos frescos por día

$$
C P C+P C / C T C=T C T
$$

Donde:

CPC Capacidad en peso por caja $10 \mathrm{~kg}$

PC Peso de la caja kg 1.34

CTC capacidad transporte carga 3500

TCT Total de cajas por transporte 
(vi) Cálculo de vehículos para la distribución alimentos última milla por año

$$
(\mathrm{CUM} / \mathrm{CPC}) / \mathrm{TCT}=\mathrm{CVa}
$$

Donde:

CUM Consumo/kg Última milla
CPC Capacidad Peso Caja
TCT Total cajas por transporte
CVa Cantidad de vehículos para la distri-
bución de alimentos frescos

Para el cálculo del valor monetario de la contaminación $\mathrm{CO} 2$ por combustión del sector transporte carga en carretera en la distribución de alimentos frescos, se consideró como supuesto que cada vehículo del parque vehicular contamina en promedio la misma cantidad. Se proponen dos enfoques para el cálculo, como se verá a continuación.

\section{ENFOQUES DE CÁLCULO}

El primer enfoque, utilizando los costos del mercado dados para la mitigación y/o las compras por emisión.

(vii) Cálculo de las emisiones por vehículo

$$
\mathrm{FVP} / \mathrm{ETC}=\mathrm{CV}
$$

Donde:

FVP Flota de vehículos País

ETC Emisión sector transporte carretera

CV Contaminación por vehículo

(viii) Cálculo de las emisiones por vehículo de carga

$$
\mathrm{FVC}^{*} \mathrm{CV}=\mathrm{CVC}
$$

Donde:
FVC Flota vehicular carga

CV Contaminación por vehículo

CVC Contaminación vehículos de carga

El incentivo económico que otorgan los diferentes programas para captura de carbono y la compra de derechos o permisos de emisión de dióxido de carbono son proxies para el costo de remoción de la contaminación del aire. A los países que no tienen ningún programa de incentivos se les proyectó, de acuerdo con el existente en el continente o similar. Para el continente americano se proyectó el incentivo para Colombia y Estados Unidos; en Europa, se proyectó para el Reino Unido y, en África, se proyectó con base en el monto propuesto por China.

(ix) Cálculo del Incentivo país base

$$
\mathrm{RME} / \mathrm{PIB}=\mathrm{RT}
$$

Donde:

RME Retribución por mitigación o emisión US\$ al año de estudio

PIB PIB per cápita

RT Retribución proporcional US\$

(x) Cálculo del Incentivo país sin programa de incentivos

$$
\mathrm{PIB} / \mathrm{RT}=\mathrm{RME}
$$

Donde:

PIB PIB per cápita del país sin programa de incentivos

RT Retribución proporcional US\$ del país del continente o similar

RME Retribución por mitigación o emisión US\$ al año de estudio 
Ahora bien, el segundo de los enfoques posibles, se consideraron los efectos de la contaminación en la salud.

Se planteó un segundo método para el análisis de la "metodología de datos globales" que proyecta los costos relacionados con los problemas de salud de las personas y el impacto en la ausencia laboral, ocasionados por la contaminación ambiental, tomando como referencia un país base que fue Alemania. La información de cada país/ciudad de estudio se compara con el costo de la contaminación por ese concepto en Alemania. Se utilizó la siguiente fórmula para el cálculo de la contaminación ambiental. De esta manera, el factor de comparación es el cociente entre las medias geométricas de las concentraciones de emisiones por km2 y por habitante de Alemania y el lugar de estudio.

(xi) Cálculo de datos globales contaminación

$$
\gamma=\left(\frac{P N B C_{A l}}{P N B C_{X}}\right) \frac{\sqrt{\frac{\left(C_{A l}\right)^{2}}{\left(A_{A l} P_{A l}\right)}}}{\sqrt{\frac{\left(C_{X}\right)^{2}}{\left(A_{X} P_{X}\right)}}}
$$

Donde:

y $\quad$ Factor de relación del costo de contaminación ambiental

PNBC Producto Nacional Bruto per cápita
A Superficie
C Emisiones
P Población

Al Alemania
- Externalidad desgaste de las carreteras por mantenimiento preventivo en la distribución de alimentos en la última milla

Consideraciones de cálculo:

a) Se utilizaron los datos de la Hoja de Balance de alimentos perecederos de la FAOSTAT, año 2013, para estimar el consumo por kilo per cápita a transportar, proyectándose a la ciudad en estudio.

b) Para el costo de mantenimiento rutinario de carreteras se tomaron como base, cuando se disponía de dicha información, datos de costo de referencia por país.

c) Se utilizó la flotilla total registrados en el país y la flotilla de carga liviana de la ciudad de estudio.

d) Se definió un recorrido por cada ciudad, tomando como parámetro dos anillos de uno y dos kilómetros y cuatro rayos de dos kilómetros para la distribución en la última milla.

e) Se asume que cada vehículo de la flota desgasta, en promedio, homogéneamente la carretera.

f) Cada viaje de distribución es sinónimo de un vehículo de transporte utilizado.

g) La retribución por mitigación o por compra de emisiones se obtuvo de Australia, Unión Europea, China y Costa Rica para los demás países se le proyecto utilizando el producto interno bruto.

(xii) Cálculo para el primer y segundo anillo y 4 rayos de $2 \mathrm{~km} \mathrm{c} / \mathrm{u}$

$$
\begin{gathered}
\left.\left(\left(2 R^{*} \mathrm{Pi}\right)+\left(R^{*} \mathrm{Pi}\right)+8 \mathrm{R}\right) * \mathrm{~d}\right)=\mathrm{TRL} \\
\mathrm{Pi}^{*} \mathrm{R} 2=\mathrm{TRKm} 2
\end{gathered}
$$

Donde:

$\mathrm{R}$ radio 
Pi $\quad 3.14$ constante matemática

d días por año recorrido 312 días

TRL Total Recorrido lineal anual

TRKm2 Total Recorrido km2

(xiii) Cálculo costo mantenimiento rutinario vial

$$
C V * R * M R=T C M V
$$

Donde:

$\begin{array}{ll}\text { CV } & \text { Cantidad de viajes por año } \\ \text { R } & \text { Recorrido } \mathrm{km} \\ \text { MR } & \text { Mantenimiento rutinario }\end{array}$

TCMV Total costo mantenimiento vial

\section{RESULTADOS}

De acuerdo con los datos estadísticos de los organismos mencionados y las diferentes fórmulas utilizadas, se elaboró la matriz como herramienta para el cálculo del costo de las externalidades propuestas tomando como año base 2013. Si bien es cierto, las externalidades son difíciles de costear por falta de la información requerida, este estudio propone desarrollar una herramienta financiera basada en costos dados en el mercado, conocido como un mecanismo flexible para estimar el valor monetario de dos externalidades asociadas a la distribución de alimentos perecederos en las ciudades, a saber, contaminación del aire por CO2 y el desgaste que contribuye al deterioro en el mantenimiento preventivo de las carreteras.

\section{Externalidad asociada con las emisiones de $\mathrm{CO} 2$ provocadas por la distribución de alimentos perecederos en la última milla}

Las emisiones producidas por $\mathrm{CO} 2$ por combustión por sector se detallan en la figura1. El sector transporte contempla carretera, ferroviario, marítimo y aéreo; sin embargo, el segmento de transporte por carretera representa de un $80 \%$ hasta un 95\% del sector transporte. En algunos países como Australia, Reino Unido e Italia, las emisiones por producción de electricidad y calefacción son las más importantes.

De acuerdo con la figura 2, en los países como Estados Unidos y China, la producción de electricidad es su principal emisor, seguida por el sector industrial. En el caso de China, el sector transporte es poco relevante, mientras que en los Estados Unidos el sector transporte es el segundo emisor de $\mathrm{CO} 2$ del país del cual el segmento carretera representa un 85\%.

La figura 3 muestra las emisiones del segmento carretera con respecto a las emisiones totales. El menor emisor del segmento transporte es China con un 7\% de la contaminación total del país. En el caso de Costa Rica, el segmento transporte-carretera es el principal emisor con un 68\% de las emisiones del país. En el resto de los países, la emisión del segmento transporte-carretera oscila entre un $20 \%$ y un $39 \%$.

Estos comportamientos normalmente están relacionados con las actividades económicas de los países. En el caso de China, su principal actividad económica es la industria entre las que se destacan el acero, el cemento, los químicos, los cuales demandan que la producción de electricidad sea muy importante basada fuertemente en el carbón y ese es su principal emisor de contaminación. 
FIGURA 1

EMISIONES DE CO2 POR COMBUSTIÓN POR SECTOR (2013)

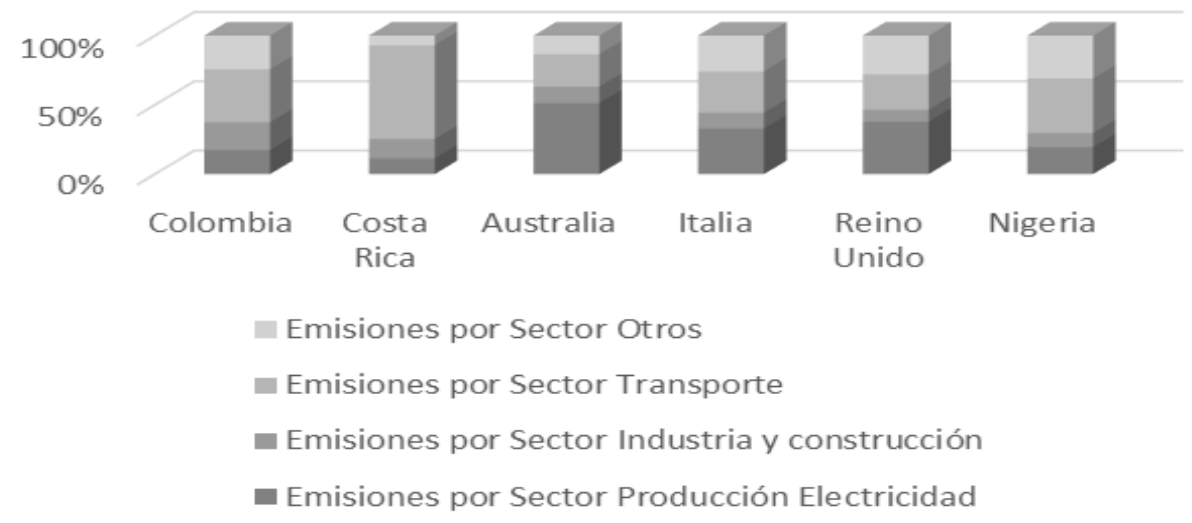

Fuente:Elaboración propia con información IEA, edición 2015

FIGURA 2

EMISIONES POR COMBUSTIÓN 2013

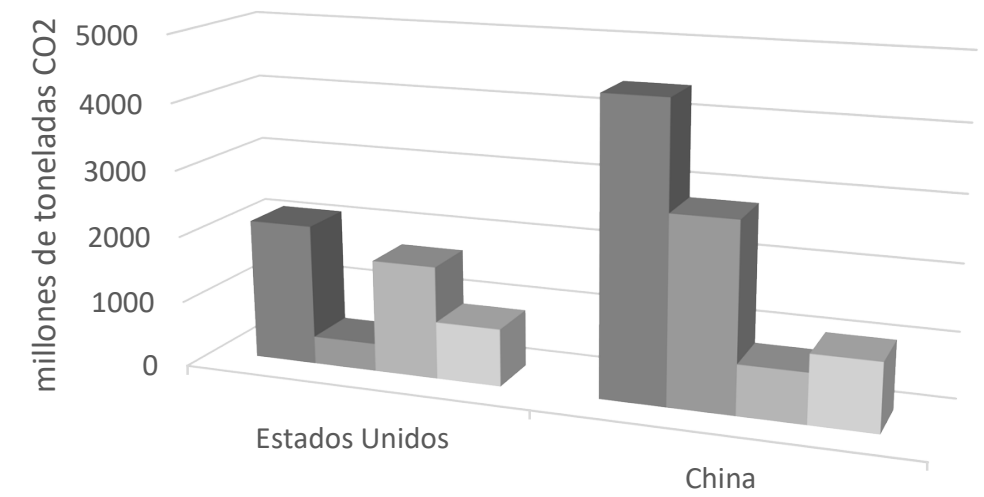

- Producción Electricidad Industria y construcción $\quad$ Transporte Otros 
FIGURA 3

EMISIONES SECTOR CARRETERA/TOTALES(2013)

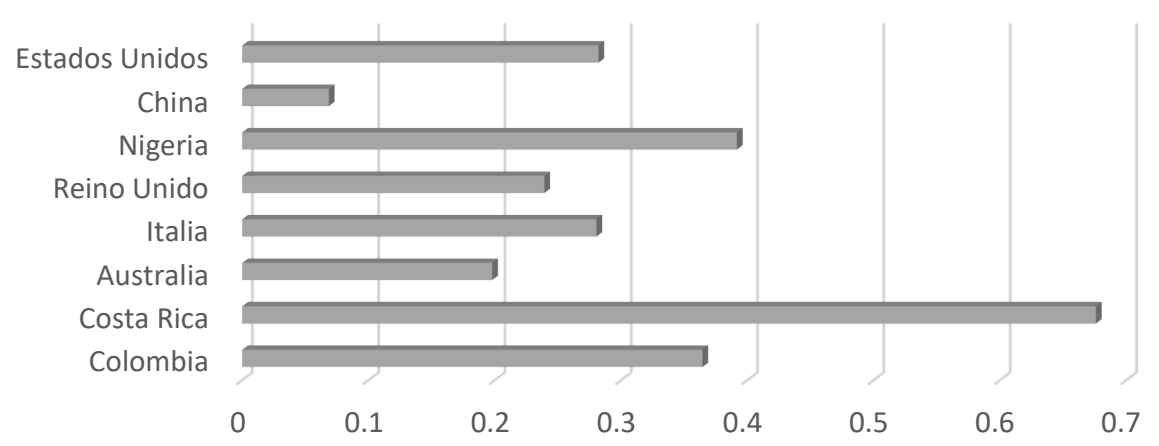

Porcentaje contaminación $\mathrm{CO} 2$ sector carretera con respecto a las

emisiones totales

Fuente: elaboración propia con información IEA, edición 2015.

En el caso de Costa Rica, su principal actividad es el turismo y la exportación de productos tradicionales (café, banano y piña) y no tradicionales. La producción de electricidad se sustenta en energías limpias, en su mayoría, y la industria es un segmento minoritario. Así, el segmento transporte carretera es su principal contaminante. Del total de emisiones del segmento transporte-carreteras, el nivel más significativo proviene de los automóviles particulares que oscilan entre un 29\% a más de un $80 \%$ del total de las emisiones país de CO2 atribuidas a este sector.

En todos los países de estudio, los vehículos particulares son la flota de mayor número de automotores emitiendo dióxido de carbono; mientras que las flotas de vehículos de carga, en la mayoría de los países, son muy pequeñas. Esto debido a que se cuenta con otros medios de transporte tanto para pasajeros como para la movilización de productos como el ferroviario y marítimo.

En el caso de Costa Rica, la movilización de los productos hacia los puertos es por carretera. Se cuenta con un sistema ferroviario muy incipiente para que ayude con la movilización de los pasajeros y comercio, esto es la principal causa por lo que el segmento carretera representa un $68 \%$ de las emisiones. El sistema de transporte carretera de Nigeria es poco desarrollado y solo cuenta, básicamente, con las vías principales asfaltadas para el trasiego de mercadería en las ciudades principales.

La figura 4 muestra las emisiones por sector transporte carretera, carga y última milla. La flotilla de vehículos utilizados para la distribución en la última milla es tan pequeña que oscila desde un $0.01 \%$ a un $0.09 \%$ (menos de un $1 \%$ en todos los casos) de la flota de carga total. 


\section{FIGURA 4 \\ EMISIONES POR CO2 POR COMBUSTION, SECTOR TRANSPORTE CARRETERA, 2013}

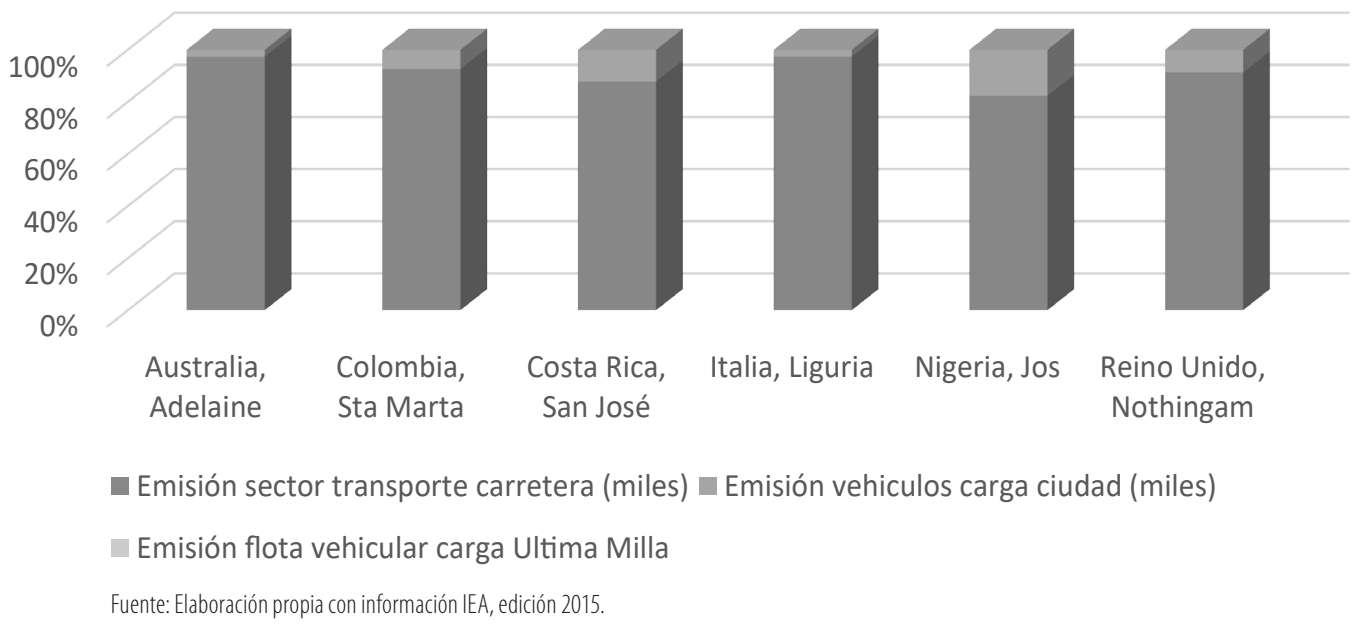

Los vehículos de carga representan de un 2\% al 20\% del segmento carretera con excepción de California, donde la flota de carga representa aproximadamente un 48\%, Baton Rouge un 38\% y Nigeria un 22\%. Este dato es un indicador de que la flota de carga para la distribución de alimentos es minoritaria en la contribución de la contaminación por $\mathrm{CO} 2$; sin embargo, tiene alguna participación que se puede mitigar.

La tabla 1 muestra los resultados del análisis que consideró: el parque vehicular de carga de cada país, estado o ciudad, la contaminación que produce la flota completa de carga, el costo del comercio europeo de emisiones, los impuestos a las emisiones y la compensación por captura de carbono, la retribución o pago por emisión de las flotillas de carga de cada país por emisiones de CO2 y la flota utilizada para la distribución de alimentos frescos en la última milla y su respectiva retribución o inversión. La retribución por captura de carbono o pagos de derechos de emisiones de CO2 en la última mi-
Ila es inferior a 1\% con respecto a la retribución o pago del transporte de carga de los países en estudio a excepción de Costa Rica que la última milla es un 1.82\% de la retribución o pago del transporte de carga.

Las inversiones por emisiones o compensaciones por mitigación son muy diferentes en cada región. Para el continente americano, se tomó el monto de carbono secuestrado o absorbido de \$7/ton capturada retribuido en Costa Rica para la mitigación de CO2 (FONAFIFO, 2013), para proyectarlo a Colombia y a los Estados Unidos de acuerdo al PIB de cada país.

La Unión Europea (REE, 2013) estableció la iniciativa del mercado de derechos de emisión que se compran y venden entre los sectores energía e Industria desde el 2005. Ese monto de compra para emisión se tomó para Italia y Alemania y se proyectó para Reino Unido.

China (REE) aprobó a inicios de 2013 una propuesta de impuesto por emisión de dióxido de carbono, el cual no ha entrado aún en vigencia. 


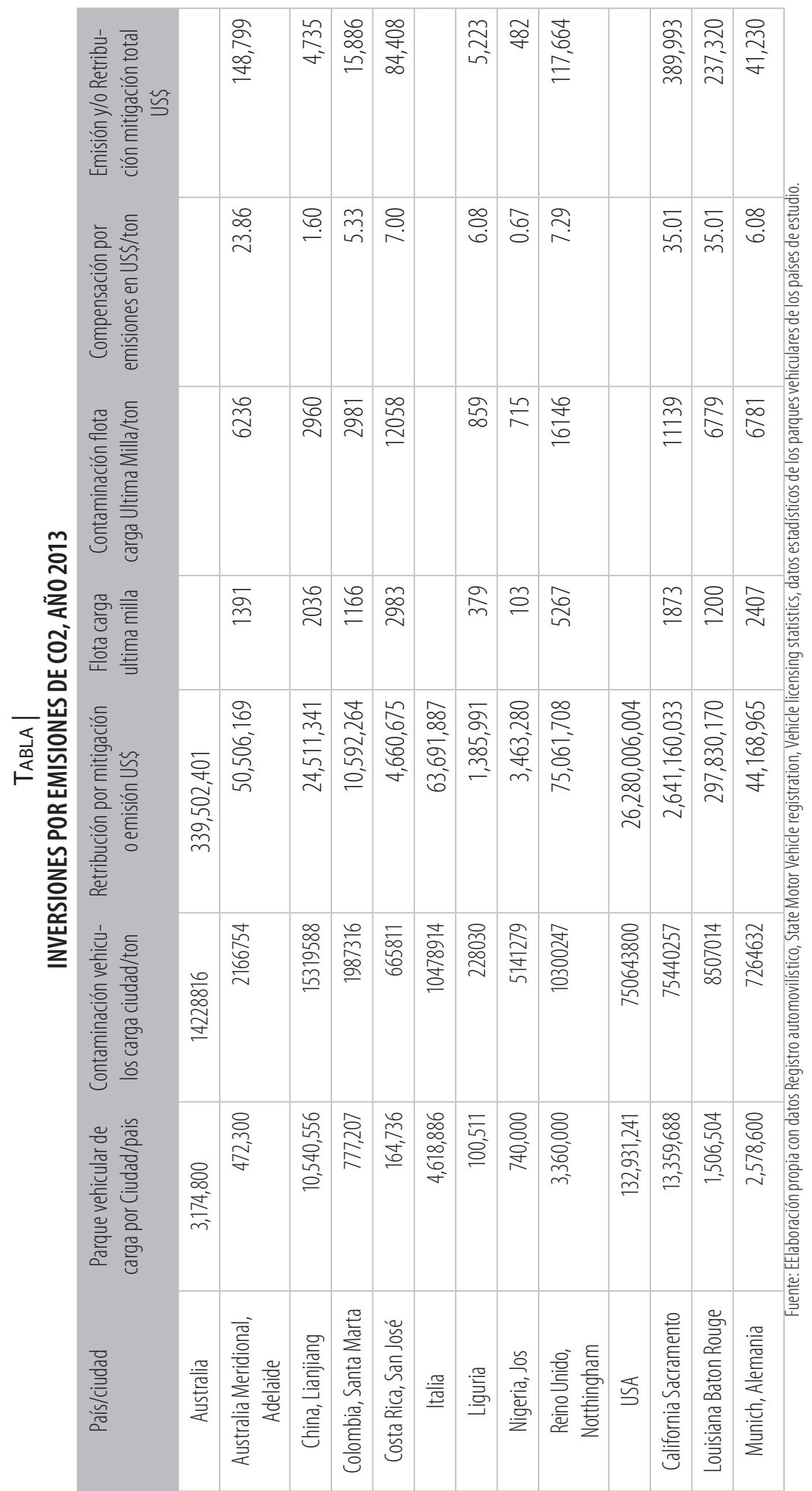


Sin embargo, se tomó la propuesta para China y proyectar Nigeria. En Australia (EFE, 2014) se utilizó un impuesto por emisiones para los industriales vigente hasta el 2012. Finalmente, se estableció la comparación del monto de inversión que correspondería por la flota de carga total en comparación con la flota de carga utilizada para la última milla la cual es insignificante.

\section{Externalidad asociada con las emisiones de $\mathrm{CO} 2$ considerando las enfermedades respiratorias y las incapacidades laborales}

La segunda metodología ofrece un enfoque de costos considerando las pérdidas de incapacidad laboral ocasionados por enfermedades respiratorias, las pérdidas de recursos humanos por muertes, los costos de rehabilitación de las personas afectadas por enfermedades respiratorias, el costo de las enfermedades cardiovas- culares, y el costo de reparación de estructuras y edificios como consecuencia de la contaminación atmosférica. En la figura 5 se plantean los resultados del modelo.

Alemania es el país base de referencia en la propuesta del instrumento. Al aplicarse la metodología con los países de estudio, el factor resultante para el 2013 fue: para Italia 2.7 veces; Reino Unido 1.3 veces; Australia 3.2 veces y Estados Unidos 1.3 veces con respecto al monto del costo de la contaminación de Alemania por US\$12 millones anuales.

Lo anterior da como resultado, para Italia, una inversión de \$32.4 millones; Reino Unido y Estados Unidos de \$15.6 millones y Australia de $\$ 38.4$ millones anuales para compensar los efectos sobre la salud y ausentismo laboral de los habitantes. Para el resto de los países los resultados del instrumento no tienen razonabilidad por cuanto el país base tiene factores de comparación muy altos.

FIGURA 5

COSTO CONTAMINACIÓN AMBIENTAL BASE ALEMANIA (2013)

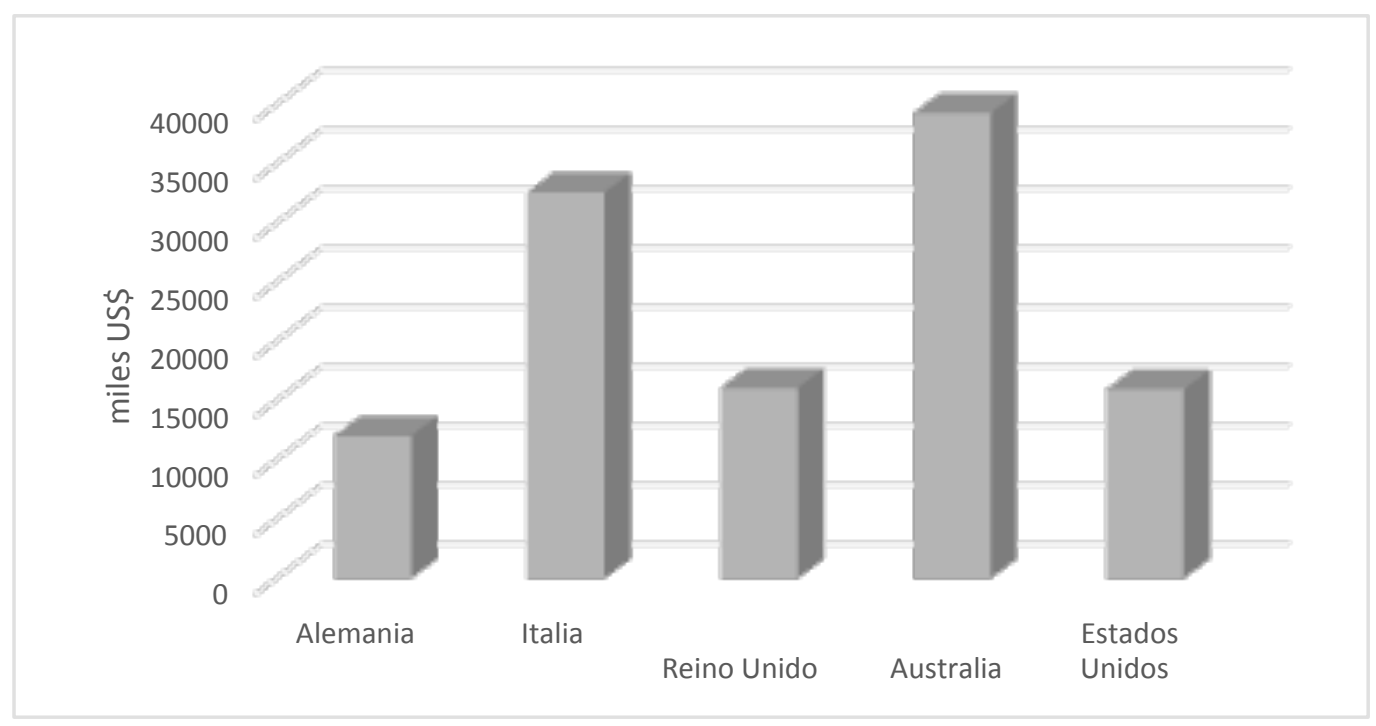

Fuente: Elaboración propia con datos de Banco Mundial, IEA, Metodología cálculo C. Díaz. 2013 


\section{Externalidad asociada con el deterioro de las carreteras provocadas por la distribución de alimentos perecederos en la última milla}

El punto de partida para este cálculo fue conocer el consumo de alimentos perecederos de cada país y su per cápita. En la figura 6 se muestra que países como Australia, Estados Unidos, Nigeria y Costa Rica no logran el consumo de 400 gramos de alimento fresco diario recomendado por la Organización Mundial de la Salud (OMS, 2015). Mientras tanto, China, Colombia, Italia y Reino Unido sí alcanzan el consumo de alimentos perecederos recomendados. Estos datos evidencian el reto de concientizar a las poblaciones de la importancia de la alimentación nutritiva y saludable para lograr una ingesta balanceada de alimentos frescos.
La figura 7 muestra el consumo diario de alimentos perecederos que serán distribuidos en la zona urbana. Esto sin tomar en cuenta las pérdidas de alimentos que ocurren a nivel de la distribución urbana, venta al detalle y hogares.

La última milla Liguria y Santa Marta son las ciudades con menor consumo de productos perecederos; mientras que Nottingham representa el mayor consumo, seguido de San José. Una vez más queda en evidencia, en los centros de las ciudades, que se debe motivar a sus habitantes a ingerir mayor cantidad de productos frescos.

La tabla 2 resume los cálculos de la externalidad desgaste de carreteras en la etapa de mantenimiento preventivo, para las ciudades de Lianjiang, San José, Liguria y Jos, por contar con información disponible para el cálculo. Para establecer una ruta para la entrega de alimentos de acuerdo con el recorrido planteado de dos anillos de uno y dos kilómetros cada uno y cuatro rayos de dos kilómetros cada uno, se estimó

FIGURA 6

CONSUMO ALIMENTOS FRESCOS PER CÁPITA (2013)

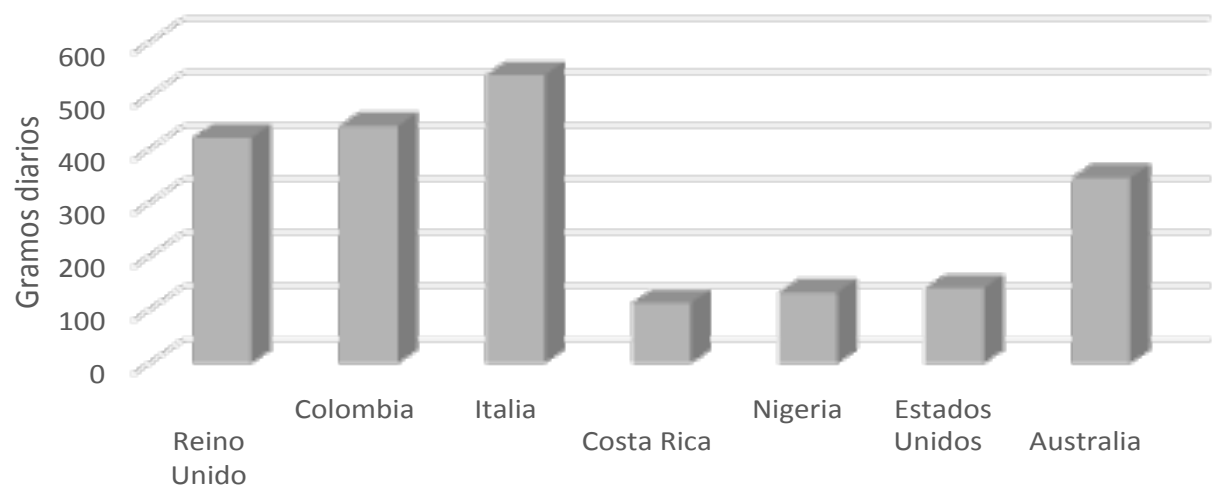

Fuente: Elaboración propia con datos de consumo per cápita de la FA0, 2013. 


\section{FIGURA 7 \\ CONSUMO DIARIO \\ ULTIMA MILLA, 2013}

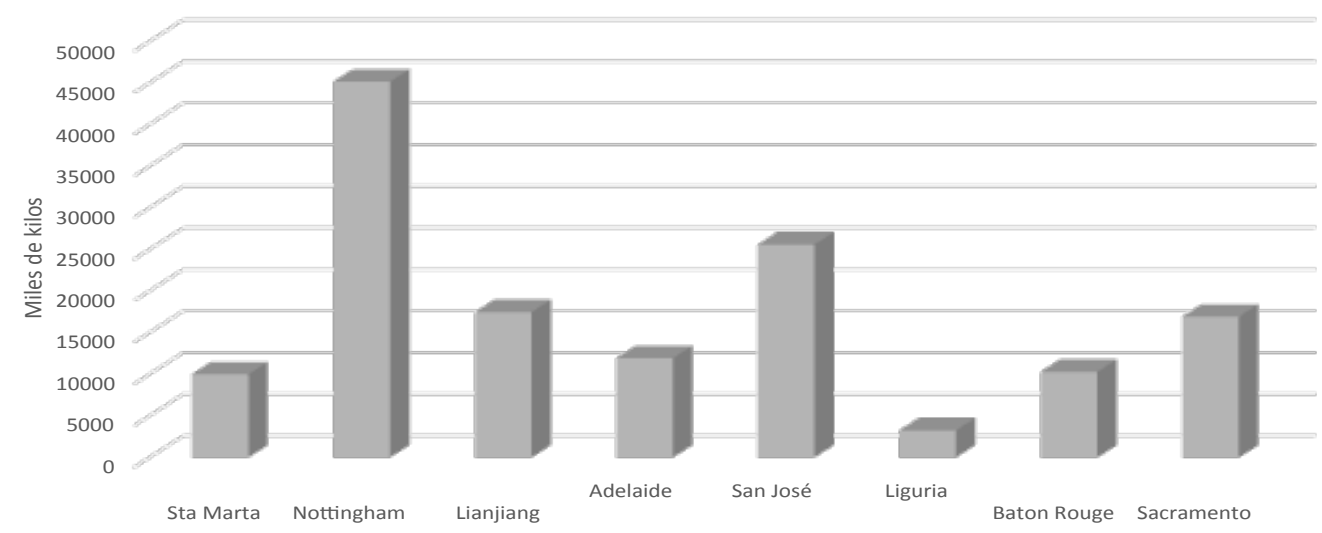

Fuente: Elaboración propia con datos de población y consumo per cápita diario 2013.

TABLA 2

RESUMEN POR CIUDAD DEL COSTO MANTENIMIENTO PREVENTIVO, AÑ0 2013

\begin{tabular}{|l|l|l|l|l|}
\hline \multicolumn{1}{|c}{ Detalle/Ciudad } & \multicolumn{1}{c}{ Lianjiang } & \multicolumn{1}{c|}{ San José } & \multicolumn{1}{c|}{ Liguria } \\
\hline Cantidad de viajes anuales & 2036 & 2983 & 379 & 103 \\
\hline Recorrido km & 13541 & 8374 & 8374 & 8374 \\
\hline Mantenimiento rutinario US\$/km & 7486 & 10110 & 6866 & 2291 \\
\hline Total costo mantenimiento por desgaste US\$ & 101366.429 & 84665.609 & 57497.404 & 19185.017 \\
\hline
\end{tabular}

Fuente: Elaboración propia con datos de FAO, Banco Mundial, Road Maintenance BM.

que el recorrido oscila entre los 21 y los 43 kilómetros por día, dependiendo de cada ciudad.

Con la información existente se requirió, en algunos casos, proyectar el monto de mantenimiento al 2013 aplicando la inflación hasta esa fecha (BM-TRN4) y se calculó el monto aproximado de inversión en mantenimiento preventivo para las carreteras en la ciudad. Si los distribuidores de alimentos para estas ciudades recorren la misma cantidad de kilómetros en San José, Liguria y Jos, y las inversiones por de- terioro oscilan desde los 20 hasta los 85 millones de dólares, es debido al costo del mantenimiento preventivo en cada país por kilómetro.

Asimismo, una ciudad como Lianjiang, tiene el mayor consumo per cápita (un 52\% más que Italia), mayor población (un 25\% mayor que Liguria), mayor cantidad de viajes y kilómetros que se recorren (un 38\% más que el resto de las ciudades), su costo de mantenimiento es menor (un 26\% menos que San José) y su inversión 
es solo un 16\% mayor a San José. Este fenómeno es lo que establece una gran diferencia en la eficiencia de los recursos financieros para el mantenimiento y construcción de la red vial de cada país. De acuerdo con un estudio realizado por la Organización para la Cooperación y el Desarrollo Económicos (OCDE), desde el 2009 los países han venido disminuyendo los montos de inversión hasta en un 30\% en el mantenimiento de las carreteras.

\section{ANALIZANDO LOS RESULTADOS}

El modelo financiero de costos propuesto se basa en supuestos cuyas variaciones podrían arrojar resultados numéricos diferentes. Sin embargo, ofrece una alternativa viable de cálculo utilizando la información existente en cuanto a costos conocidos e utilizados por el mercado que nos llevan a lograr el costo meta.

Para los cálculos del costo de la contaminación (CO2 emitido por el sector transporte de carga para la distribución de alimentos), el rango a utilizar era muy específico pues incluyó solo la contaminación por CO2 del segmento transporte de carga usado para la distribución de perecederos en las ciudades y las estadísticas de las flotillas vehiculares de los países y sus modalidades.

Así, se estimó la flota de carga en la última miIla, estableciendo como supuesto que la contaminación de los vehículos es uniforme. En los países en donde se conozcan las características de la flota de carga, en cuanto a edad promedio vehicular, tipo de combustible utilizado y conglomeraciones viales, se minimiza el margen de error en el cálculo de la emisión de este segmento. Sin embargo, para este estudio no se contó con dicha información.

El costo asignado a una tonelada emitida o mitigada de CO2 en el año 2013 es muy diverso. En el caso de la Unión Europea, se utilizaron los precios de mercado de derechos de contamina- ción, así como el precio de captura de carbono pagado en Costa Rica para la proyección de los países del continente americano considerados en este estudio. También, se incorporó el impuesto de emisión de Australia y el impuesto propuesto por el gobierno de China como insumo base para proyectar Nigeria. Esta es la información existente de precios vía impuestos, precios de compra de emisión y retribuciones por mitigación utilizadas en esta metodología de costos.

En relación con el método propuesto, se creó una matriz en la que se agruparon los diferentes datos numéricos existentes al año 2013, por país de estudio, en el tema de emisiones por sector, flota vehicular por sector, emisiones por vehículo, emisiones de la flota de vehículos de carga, emisiones de la flota utilizada para la distribución de alimentos frescos y, finalmente, el precio o inversión por tonelada en dólares americanos y el monto total proyectado de la inversión para compensar esta externalidad.

En cuanto a los resultados obtenidos al calcular el costo de la externalidad de las emisiones de CO2 del sector transporte de carga para la distribución de alimentos, el país que debería realizar una mayor inversión son los Estados Unidos por su flota total vehicular (debería invertir US\$50,585 millones). En US, el sector transporte es el segundo sector más contaminante después del productor de electricidad y calefacción.

En cuanto al segmento vehículos de carga, el costo representa en promedio un 10\% para el estado de California y un 1\% para el estado de Louisiana en relación con el total del transporte carretera y la inversión en la última milla es menor al 1\% del total del transporte de carga. Finalmente, el país con menor inversión es Nigeria, con US\$16 millones para el total del sector transporte carretera, la inversión para el sector carga es de 22\% y para el transporte utilizado en la última milla la inversión es insignificante. Los resultados van acorde a los países y sus flotas vehiculares. 
En el caso de China, el país más contaminante de los estudiados, las fuentes de mayor contaminación por $\mathrm{CO} 2$ son el sector productor de electricidad (49\%) y el sector industrial y construcción (31\%) no así el sector transporte que representa un 7\% del total de emisiones de CO2. La Tabla 3 muestra los valores finales de la metodología de costos utilizada para la externalidad emisiones de CO2 por combustión.

La flota total vehicular de Australia debería reconocer una inversión de US\$ 1.8 millones por captura de carbono, la de China US\$ 988 millones por mitigación y Reino Unido por US\$782 millones. Los pagos por emisión o mitigación de carbono del segmento transporte- carga representan de un 2\% a un 21\%. La flotilla de carga del estado de California, en los Estados Unidos, es la de mayor inversión por US\$2.641 millones, seguida por la flota de carga de Louisiana con US\$297 millones. La flota de la última milla tiene el mismo comportamiento siendo las flotas de Sacramento y Baton Rouge las que muestran una mayor inversión; sin embargo, no alcanzan ni un $1 \%$ con respecto a la inversión de los segmentos de carga.

Los datos que nos muestra esta herramienta evidencian los retos del sector distribuidor para mejorar la eficiencia logística en el transporte de alimentos nutritivos en las ciudades. Aunque las cifras totales de los parques vehiculares en carretera son en algunos casos importantes, no se puede perder de vista que esos costos son muy moderados en comparación a los segmentos de manufactura y producción de electricidad.

En el caso de que se tomaran los datos del transporte de carga, los resultados son menores a un 1\% del total. Por ende, los vehículos de carga para la última milla son poco atractivos; sin embargo, existen y se deben considerar para planes de acción dentro del sector agropecuario para la mitigación de la contaminación por $\mathrm{CO} 2$.

La mayor ventaja de este enfoque es que ilustra los posibles costos que se producen en el último eslabón de la cadena alimenticia debido a la distribución de alimentos frescos en las

TABLA 3

COSTOS DE INVERSIÓN POR EMISIÓN DE CO2 SECTOR TRANSPORTE CARRETERA US\$, 2013

\begin{tabular}{|l|r|r|r|}
\hline \multicolumn{1}{|c|}{ País/ciudad } & $\begin{array}{c}\text { Costo por emisión o pago } \\
\text { por mitigación Flota } \\
\text { vehicular carretera }\end{array}$ & $\begin{array}{c}\text { Costo por emisión o pago } \\
\text { mitigación vehiculos carga }\end{array}$ & $\begin{array}{c}\text { Costo por emisión o pago } \\
\text { mitigación vehiculos } \\
\text { U Milla }\end{array}$ \\
\hline Nigeria, Jos & 16.099 .574 & 3.463 .280 & 299 \\
\hline Costa Rica, San José & 33.600 .000 & 4.660 .675 & 84.408 \\
\hline Australia, Adelaine & 1.837 .235 .400 & 50.506 .169 & 148.799 \\
\hline USA Louisiana & 50.585 .884 .612 & 297.830 .170 & 237.320 \\
\hline Munich, Alemania & 897.408 .000 & 44.168 .965 & 41.230 \\
\hline Colombia, Santa Marta & 132.715 .395 & 10.592 .264 & 15.886 \\
\hline Reino Unido, Notthingham & 782.663 .511 & 75.061 .708 & 117.664 \\
\hline USA California & 50.585 .884 .612 & 2.641 .160 .033 & 389.993 \\
\hline Italia, Liguria & 576.811 .690 & 1.385 .991 & 5.223 \\
\hline China, Lianjiang & 988.320 .000 & 24.511 .341 & \\
\hline
\end{tabular}

Fuente: Elaboración propia con datos de IEA, Parlamento Mercado Europeo, Fonafifo 
ciudades. Los resultados del modelo Costos de Externalidades, que considera los efectos en la salud humana, ausentismo laboral y deterioro en las estructuras física, muestran que el mecanismo de comparación con un país base. De acuerdo con la tabla 4, la comparación del costo de la contaminación de Alemania tuvo resultados coherentes en países como Italia, Reino Unido, Australia, Estados Unidos.

También, se aplicó la metodología a las ciudades de estudio con Munich como ciudad base y considerando solo la contaminación del sector transporte carretera. Los resultados fueron: Lianjiang 1.3 veces (US\$ 312 mil); Adelaide 3 veces (US\$ 713 mil); Sacramento 3 veces (US\$
706 mil) y Baton Rouge 1.6 veces (US\$ 374 mil) con respecto al costo de la contaminación de Munich por US\$ 237 mil. Los resultados de los factores numéricos de las otras ciudades fueron muy dispersos, por lo que se perdió la esencia del modelo.

Los resultados obtenidos de la externalidad por desgaste de carreteras muestran que las inversiones finales oscilan en un rango de US\$20 a US\$100 millones debido, principalmente, al costo del mantenimiento preventivo de cada país y a la cantidad de kilómetros que se debería atender con este servicio. Los montos son muy superiores a las inversiones por la conta-

TABLA 4

COSTO CONTAMINACIÓN AMBIENTAL CON BASE ALEMANIA, 2013

\begin{tabular}{|c|c|c|c|c|c|}
\hline \multicolumn{1}{|c|}{ País } & Alemania & \multicolumn{1}{c}{ Estados Unidos } & \multicolumn{1}{c|}{ Reino Unido } & Italia & Australia \\
\hline Costo contaminación & 12058 & 15980 & 16087 & 32601 & 39294 \\
\hline
\end{tabular}

Fuente: Elaboración propia.

\section{FIGURA 8 \\ COSTO DEL MANTENIMIENTO PREVENTIVO 2013}

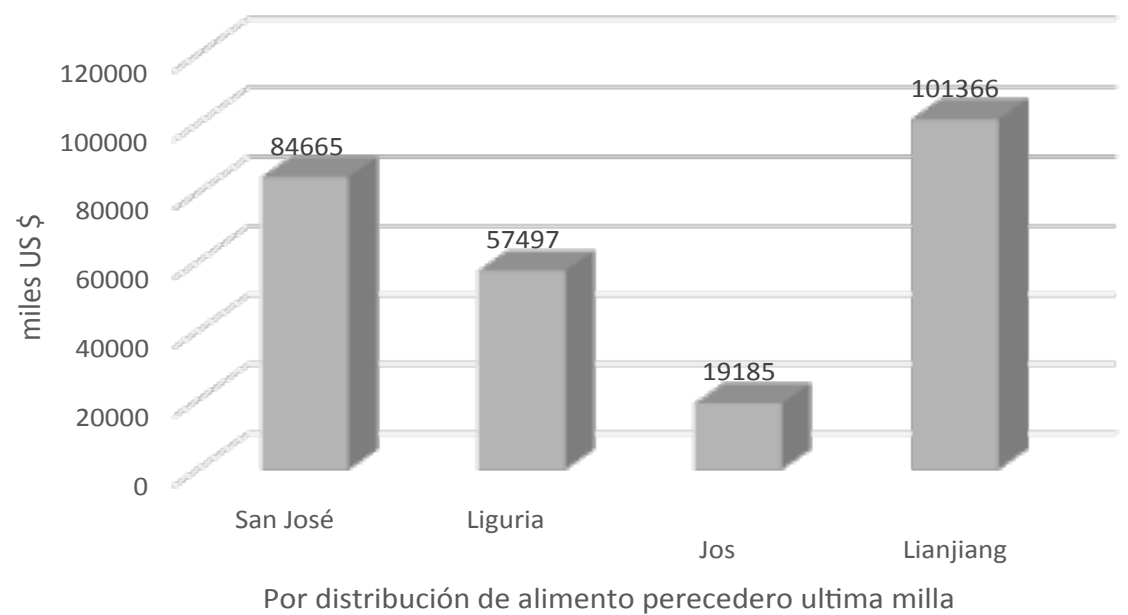

Fuente: Elaboración propia. 
minación por CO2 y las razones son un tema para discutir.

Existe la probabilidad de que los costos del mantenimiento de carreteras sean más conocidos, pero eso no quiere decir que sean los correctos, por las distorsiones que se dan en los países en este tipo de contrataciones. En Europa, por ejemplo, un kilómetro de carretera puede costar miles de dólares mientras que, en Latinoamérica, millones de dólares y, por ende, el mantenimiento preventivo puede ser muy elevado para algunos países.

Esta propuesta financiera de costos fue la mejor alternativa para crear escenarios de los costos de estas externalidades. Tiene la ventaja de que se proyecta a partir de la información de costos conocidos y permite el acercamiento a una proyección de un dato basado en información existente y puede sensibilizarse. Su desventaja es la precisión de los supuestos que se consideran en la proyección de los costos, al igual que otras metodologías prevalentes hasta ahora tienen un nivel de incertidumbre similar.

\section{CONCLUSIONES}

Los objetivos propuestos de analizar las externalidades en la distribución de alimentos perecederos en la última milla y crear una herramienta financiera de costos para estimar la o las externalidades encontradas cuantificables se lograron con la información existente, realizando proyecciones y estableciendo algunos supuestos. La distribución de alimentos perecederos debe ser muy eficiente, ya que los productos frescos tienen un ciclo de vida corto $y$, dentro del marco de la distribución de mercaderías de todo tipo, su participación es muy pequeña. Sin embargo, también contribuye a la generación de externalidades negativas y, por ello, es importante la visión de considerar que toda actividad personal o empresarial tiene su contribución a estos costos poco reconocidos.
Las externalidades son un tema clave para disminuir las distorsiones del mercado, desde tiempo atrás ya se conocía la existencia de estos costos; sin embargo, no se le había dado la importancia que tiene en la actualidad. Por ello, se busca crear consciencia y motivar la construcción de información requerida para el cálculo preciso de las externalidades.

La herramienta usada para el cálculo de los costos de estas dos externalidades no solo representa una propuesta de estimación de costos, sino una puerta al diálogo y a la reflexión en el sector de alimentos perecederos acerca de este costo poco reconocido y no asociado a la actividad como tal. Es un hecho que su reconocimiento ayuda a la generación de información de mayor calidad para tener cálculos más exactos y cambios que generen una contribución para el medio ambiente.

Esta propuesta es un insumo, un instrumento para que los participantes del mercado reconozcan el impacto de su actividad con costos de las externalidades y puedan trabajar en las mejoras de los procesos y las técnicas para contribuir con la mitigación de las mismas. Existen muchas limitantes para el cálculo y a la vez muchos modelos propuestos; sin embargo, la desventaja de todos ellos radica en los supuestos debido a la falta de información de calidad. Esto no quiere decir que no se pueda partir de la información con la que se cuenta e iniciar el proceso de crear y mejorar la información existente sino, al contrario, lo medular es trabajar sobre este principio.

Un reto importante que evidencia el estudio no solo es la falta de información relevante para el costeo, sino la desactualización de los datos existentes en muchos países, y esto obliga a los autores de las bases de datos a realizar proyecciones estadísticas. En este estudio se utilizó esta técnica para lograr acercarse a datos más actualizados. 
En cuanto a los resultados del estudio, en Costa Rica, Italia, China y Nigeria se obtuvo información para el cálculo de las dos externalidades propuestas, mientras que la externalidad de contaminación por combustión de $\mathrm{CO} 2$ se logró calcular en las nueve ciudades estudiadas. Se actualizó alguna información al año 2013, a través del índice de inflación, proyectando los datos para todos los países y así estimar el costo de la externalidad de la contaminación atribuida a la distribución de alimentos perecederos en la última milla.

En cuanto al costo del mantenimiento preventivo en las carreteras, Costa Rica es el país con mayor costo $\$ 10 \mathrm{mil} / \mathrm{km}$, China sigue con $\$ 7$ $\mathrm{mil} / \mathrm{km}$, Italia con $\$ 6 \mathrm{mil} / \mathrm{km}$ y Jos presenta el costo más bajo con $\$ 2 \mathrm{mil} / \mathrm{km}$, para un recorrido igual de 8 mil km/año. En San José, Liguria y Jos las inversiones por mantenimiento preventivo son radicalmente diferentes a causa de los costos de mano de obra, gastos de asignación de la obra, los procesos lentos y engorrosos, costo de los materiales, entre otros, que encarecen el monto de la obra en cada país.

Costa Rica invertiría un 32\% más de recursos por el mismo recorrido que Italia y, en Lianjiang, teniendo un recorrido de 5 mil kilómetros adicionales, la inversión es solamente un 16\% mayor a la de Costa Rica. Los costos de mantenimiento en Nigeria están ligados no solo a un costo más bajo de la mano de obra, sino también a los estándares de calidad y materiales utilizados y la mayor parte de la red vial es lastrada (carreteras no pavimentadas).

Nottingham y San José son las ciudades que requieren la mayor cantidad de viajes de camiones para la distribución de alimentos en la última milla y este evento está vinculado a la población existente en ella y al consumo per cápita de cada ciudad. Nottingham, con más de 100 mil habitantes, y San José, con 84 mil habitantes, son las que tienen la mayor pobla- ción en la última milla y las ciudades con menor población en la última milla son Jos y Liguria.

Sin embargo, San José es la ciudad con menor consumo per cápita de alimentos perecederos (113 kg), seguida por Adelaida (127 kg) y Lianjiang, la de mayor consumo per cápita (411kg) seguida por Liguria con 198 kg. Esta información es un indicador que muestra las tendencias de comportamientos alimenticios de cada población y los requerimientos de alimentos frescos que consumen para satisfacer sus necesidades, así como el reto para estimular el consumo apropiado de los alimentos nutritivos de parte de las autoridades competentes en la materia.

En cuanto a la contaminación de CO2 emitida por los vehículos que distribuyen alimentos frescos en la última milla, es un criterio muy innovador por tratarse de un cálculo tan específico en una actividad en particular. En la mayoría de los países no se dispone de información actualizada de sus flotillas vehiculares particulares, de carga, autobuses, clasificado por modelo, cilindraje, tipo combustible y revisiones anuales. Este vacío debería ser una llamada de atención a los países para recopilar este tipo de información para ayudar a lograr un costeo de alta calidad.

El conocimiento de cuántos camiones transportan solamente alimentos perecederos hacia las zonas urbanas es nulo, por lo que se puede trabajar con la capacidad promedio de un camión permitido en la ciudad y estimar la cantidad de producto de acuerdo a su capacidad y a la demanda de alimentos en la última milla. Así, se podrá proyectar la cantidad de vehículos necesarios, lo que contamina cada vehículo y lo que se debería invertir para mitigar esas emisiones. El punto es que, aunque no se cuente con toda la información, se puede estimar un aproximado y tender un primer puente que concientice lo que contamina cada actividad 
productiva o de servicios y se planteen alternativas de mitigación viables en todos los sectores de la sociedad.

China es uno de los países más contaminantes a nivel global, pero en el sector de carreteras y de carga en carretera el nivel de contaminación es muy bajo (7\% con respecto al total país). Por ello, en este estudio China no es abanderado en la contaminación por la distribución de alimentos en carretera; no así los Estados Unidos que cuenta con una flota vehicular que contribuye en las emisiones como el segundo foco emisor de contaminación generada en el país, por lo que la inversión en mitigación de las emisiones es muy alta evidenciando la urgencia de medidas de captura de carbono. Sin embargo, ese no es el panorama para la distribución en la última milla, es poco relevante en comparación a la flota total vehicular. Por otro lado, China está en un proceso, creando iniciativas para mitigar sus emisiones, que producen daños como país a nivel interno, desde la salud hasta la mortalidad e impactando al mundo entero.

Todos los productores de emisiones en menor o mayor cantidad no las pueden sostener para sí, sino que son para perjuicio del mundo entero, así es como todos contribuimos con dañar el planeta, por ello el primer paso es concientizarse de esta realidad. Al delimitar el estudio a la última milla, las inversiones por emisiones en la actividad de distribución de alimentos frescos son muy pequeñas comparadas con el segmento de carga total o flotilla vehicular total del país, pero como parte del mercado productor contribuyente en las emisiones se puede participar en la mitigación de las mismas.

\section{REFERENCIAS}

Asociación Colombiana de vehículos automotores (ANDEMOS). (s.f.). Cifras y estadísticas (2013). Recuperado de http://www.andemos.org/index. php/cifras-y-estadisticas-2/
Australian Bureau Statistics (ABS). (2013). Motor Vehicle Census. Retrieved from http://search.abs.gov.au/s/ search.html?cluster 1=registered + motor+vehicles\&clicked_fluster=total+registered+motor+vehicles\&cluster $0=$ motor+vehicles\&form $=$ simple\&origin $=41.833298 \% 2$ C $12.883301 \&$ profile $=\_d e-$ fault\&query $=\% 60$ Total+registered +motor+vehicles\%60\&collection=abs\&f.Date\%7Cd=d\%3D2013

Azqueta, D. (2002). Introducción a la Economía Ambiental. Madrid, ES: McGraw-Hill.

Banco de la Republica de Colombia (BRC). (s.f.). Meta de Inflación. Recuperado de http://www.banrep. gov.co/es/precios/meta-inflacion

Banco Mundial. (s.f.). Datos. Emisiones de $\mathrm{CO} 2$ toneladas métricas per cápita 2013. Recuperado de http://datos.bancomundial.org/indicador/ NY.GDP.PCAP. CD? locations=GB

Banco Mundial. (s.f.). PIB per cápita US\$, 2013. Recuperado de http://datos.bancomundial.org/indicador/EN.ATM.CO2E.PC?locations=GB.

Banco Mundial. (s.f.). Población Total, 2015. Población urbana, 2015. Recuperado de https://datos.bancomundial.org/indicador/SP.POP.TOTL.

Burningham, S. \& Stankevich, S. (2005). Por qué el mantenimiento vial es importante y cómo hacerlo realidad. Transport Note No.TRN-4. Whashigton, DC: Banco Mundial. Recuperado de http:// documentos.bancomundial.org/curated/ es/211191468323695241/pdf/339250BRIOFREN00Box379836B00PUBLIC0.pdf.

Central Bank of Nigeria (CBN). (s.f.). Data statistics inflation rates. Retrieved from http://www.cbn.gov. ng/rates/inflrates.asp?year=2013

China Statistical YearBook. (s.f.). Transport, Postal and Telecommunication Services. Retrieved from http:// www.stats.gov.cn/tjsj/ndsj/2013/indexeh.htm

Delacámara, G. (2008). Guía para decisores Análisis económico de externalidades ambientales. Comisión Económica para América Latina (CEPAL): Colección Documentos de proyectos. Publica Naciones Unidas. New York, NY: Naciones Unidas. 
Díaz. C. (2005). Metodología para la evaluación de los costos de la movilidad en el transporte público-Cálculo de Externalidades. Medellin, CO: Universitat Politécnica de Catalunya.

Food and Agricultural Organization of the United Nations (FAO). (s.f.). Balance Sheet, Fresh fruits \& vegetables. (FAOSTATS, 2013) Crops Q. Production, Importation \& Exportation, Fresh vegetables \& fruits. Retrieved from http://www.fao.org/faostat/en/\#data/QC

Global rates (WID). (s.f.). Datos históricos de la inflación de China. Recuperado de http://es.global-rates.com/ estadisticas-economicas/inflacion/2013.aspx

Gobierno de Australia. (s.f.). Australia deroga su impuesto al CO2. EFE: verde (2014). Recuperado de https://www.efeverde.com/noticias/australia-deroga-el-impuesto-las-emisiones-de-carbono/

Inflation.eu (WID). (s.f.). Inflation Data Germany. Retrieved from http://es.inflation.eu/tasas-de-inflacion/alemania/inflacion-historica/ipc-inflacion-alemania.aspx

Instituto Nacional de Estadística y Censo (INES). (s.f.). Costa Rica: Vehículos Automotores en Circulación, según estilo. Recuperado de http://www.inec. go.cr/documento/cuadro-951-costa-rica-vehiculos-automotores-en-circulacion-segun-estilo-2012-2015

International Energy Agency (IEA). (s.f.). CO2 Emission from fuel combustion (2013). Retrieved from https://www.iea.org/publications/freepublications/publication/CO2EmissionsfromFuelCombustionHighlights2017.pdf

Italian National Institute of statistics (ISTAT). Vehicles by vehicle type and year (Public Register of Motor). Retrieved from http://www.istat.it/en/liguria/ data?qt=gettable \&dataset=DCIS_VEICOLIPRA\& $\operatorname{dim}=16,4,0,0$ \&lang $=1 \&$ tr $=0 \&$ te $=0$

Menéndez, J. (2003). Manual Técnico Mantenimiento de caminos con microempresas. Lima: Oficina Internacional del Trabajo. Recuperado de http:// www.ilo.org/public/spanish/employment/recon/eiip/download/mcrmantec.pdf
Menéndez, J. (2012). Carreteras nos cuestan un Pastón. Revista Tráfico y Seguridad Vial, (212), 13-15. Recuperado de https://issuu.com/buenaspracticasmotos/docs/revista-tra_fico-y-seguridad-vial-

Ministerio de Ambiente y Energía (MINAE) Fondo Nacional de FinanciamientoForestal (FONAFIFO). (2013). Servicios ambientales para captura de carbono. Costa Rica. Recuperado de http://www. fonafifo.go.cr/psa/

Municipalidad de Santa Marta. (s.f.). Plan de DesarroIlo, 2012-2015. Colombia. Recuperado de http:// www.santamarta.gov.co/portal/

Organización Mundial de la Salud (OMS). (2015). Alimentación Sana. Nota descriptiva No. 394. Recuperado de http://www.who.int/mediacentre/ factsheets/fs394/es/

Organization for Economic Co-operation and Development (OECD). (2013) International Transport Forum. (1995-2011). Spending on Transport Infrastructure. Retrieved from https://www.itf-oecd.org/ sites/default/files/docs/13spendingtrends.pdf

Pigou, A. (1920). The Economics of Welfare. UK: Palgrave McMillan. doi: 10.1057/978-1-137-37562-9.

Republica Empresa y Economía. (2013). China crea un impuesto sobre el carbono. Ecología verde. Recuperado de http://empresayeconomia.republica. com/desarrollo-sostenible/china-crea-un-impuesto-sobre-el-carbono.html

Republica Empresa y Economía. (2013). Europa retira 900 millones de permisos de emisiones de $\mathrm{CO} 2$. España: El país. Recuperado de http://empresayeconomia.republica.com/desarrollo-sostenible/europa-retira-900-millones-de-permisos-de-emisiones-de-co2.html

Reyes, R., Galván, L., \& Aguiar, M. (2005). El precio de la contaminación como herramienta económica e instrumento de política ambiental. Revista Interciencia, 30(7), 436-441. Recuperado de http://www. scielo.org.ve/scielo.php?script=sci_arttext\&pi$d=$ S0378-18442005000700010\&lng=es\&tIng=es.

Rodrigue, Claude, \& Slack. (2013). The Geography of Transport Systems. 3ra Edición. New York, NY: Routledge. 
Russo, F. \& Comí, A. (2011). A model for simulating urban goods transport and logistics. 20th International Symposium on Transportation and Traffic Theory (ISTTT 2013). doi: 10.1016/j.sbspro.2013.05.038.

State of California (CA), Department Motor of Vehicles. (s.f.). Registered vehicle Statistics. California, USA. Retrieved from https://www.dmv.ca.gov/portal/wcm/connect/5aa16cd3-39a5-402f-9453Od353706cc9a/official.pdf?MOD=AJPERES

Taniguchi, E. (2014). Concepts of city logistics for sustainable and liveable cities. Paper presented at the First International Conference Green Cities - Green Logistics for Greener Cities, Kyoto, JP. Retrieved from https://www.sciencedirect.com/ science/article/pii/S1877042814054718.

U.S. Department of Transportation (USDT). (s.f.). Federal Highway Administration, Office of Highway Policy Information. State motor vehicles registration.
Retrieved from https://www.fhwa.dot.gov/policyinformation/statistics/2013/mv1.cfm

United Nations (ONU). (s.f.). Population data (UNSTATS, 2013). Retrieved from: https://unstats.un.org/ unsd/demographic-social/products/dyb/index. cshtml\#censusdatasets.

Vázquez, V. (2014). Externalidades y medioambiente. Revista Iberoamericana de Organización de Empresas y Marketing (Ibemark), (2), 1-15. Recuperado de: www.eumed.net/rev/ibemark/02/ medioambiente.html.

Recibido: 8 de agosto de 2017

Aceptado: 15 de febrero de 2018 